\title{
Efecto del pH en la selectividad y coagulación de los ácidos húmicos en presencia de $\mathrm{Al}^{3+}$ por potencial zeta
}

\section{Effect of $\mathrm{pH}$ on the selectivity and coagulation of humic acids in the presence of $\mathrm{Al}^{3+}$ by zeta potential}

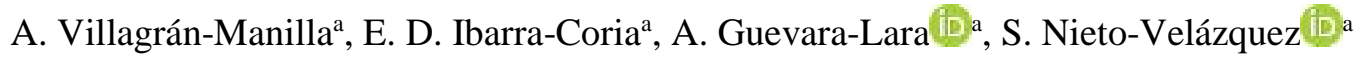 \\ a Área Académica de Química, Universidad Autónoma del Estado de Hidalgo, 42184, Pachuca, Hidalgo, México.
}

\begin{abstract}
Resumen
En esta investigación se evaluó el efecto que presenta el pH (5 y 7) en la coagulación de los ácidos húmicos (AHs) en presencia de un agente coagulante como es el aluminio $\left(\mathrm{Al}^{3+}\right)$. En el análisis fisicoquímico de los AHs en estudio (comercial AH-CH y extraídos en el laboratorio AH-A y AH-T) mostraron características poliácidas y estables, lo cual se corrobora con su capacidad de coagulación con el metal trivalente por medio de lecturas de potencial zeta. Los datos obtenidos evidenciaron que existe una rápida interacción $A H s-A l$ en un tiempo menor a 5 min y sobre todo al incrementar la concentración del metal (1, 2 y 3 mM) a un pH de 5, debido principalmente a la desprotonación de los grupos carboxílicos $(-\mathrm{COOH})$, que bajo estas condiciones pueden coordinarse, reduciendo la carga neta negativa y permitiendo así que tales complejos se aproximen e interactúen a través de puentes de hidrógeno.
\end{abstract}

Palabras Clave:

Ácido-húmico, metal, interacción, coagulación, potencial zeta.

\begin{abstract}
In this research, the effect of $\mathrm{pH}$ (5 and 7) on the coagulation of humic acids (HAs) in presence of a coagulating agent such as aluminum $\left(\mathrm{Al}^{3+}\right.$ ) was evaluated. Physicochemical analysis of studied HAs (commercial HA-CH and extracted in the laboratory HA-A and HA-T) showed polyacid and stable characteristics, which is corroborated with its ability to coagulate with the trivalent metal through zeta potential values. It was shown that there is a rapid interaction of $\mathrm{HAs}-\mathrm{Al}$ in less than 5 min and especially when increasing the concentration of the metal $(1,2$ and $3 \mathrm{mM})$ at a $\mathrm{pH}$ of 5 , due to the deprotonation of carboxylic groups (-COOH) principally, that can coordinate under these conditions, reducing the net negative charge and enabling such complexes to approach one another and interact through hydrogen bonding.
\end{abstract}

Keywords:

Humic-acid, metal, interaction, coagulation, zeta potential.

\section{Introducción}

Los ácidos húmicos (AHs) son los componentes principales y más abundantes de la materia orgánica (MO) de suelos, agua y sedimentos, se catalogan como compuestos poliméricos constituidos por monómeros o unidades estructurales (Diallo et al., 2003), de los cuales, los compuestos constituyentes más comunes son aromáticos de tipo fenólico y nitrogenados, tanto cíclicos (indol, pirimidina, purinas, entre otros) como compuestos alifáticos (aminas, amidas, esteres, monosacáridos, aminoácidos, entre otros). Los compuestos aromáticos de tipo fenólico constituyen la base o esqueleto de la molécula mientras que ciertos átomos sirven de puentes como átomos aislados: -O-, - $\mathrm{N}=$, agrupaciones elementales: - $\mathrm{NH}-,-\mathrm{CH}_{2}-$, o incluso el enlace puedeefectuarse directamente a través del carbono que une el sistema de anillos, proporcionando una estructura porosa y amorfa.
Los AHs son compuestos insolubles en medio acuoso bajo condiciones ácidas $(\mathrm{pH}<2)$ pero son solubles a valores de $\mathrm{pH}$ mayores, presentan una carga negativa y su color en solución es café (Novák et al., 200; Almendros, 2008). Todas las características que se acaban de mencionar se deben principalmente a la gran cantidad y variedad de grupos funcionales que posee su estructura sobre todo carboxílicos y fenólicos (-COOH y -OH). Sin embargo, en la actualidad es más importante caracterizar y cuantificar la cantidad de estos grupos existentes en la macromolécula, que conocer exactamente la estructura química, y esto se debe principalmente al constante cambio que presentan en el proceso de humificación que se lleva a cabo de diferentes maneras, según el tipo de microorganismos presentes, las condiciones ambientales e incluso el tipo de suelo con el que se esté tratando (Aguilera, 1989; Mosquera et al., 2007).

\footnotetext{
*Autor para la correspondencia: nieto@uaeh.edu.mx

Correo electrónico: Vi317436@uaeh.edu.mx (Abril Villagrán-Manilla), b323145@uaeh.edu.mx (Eduardo D. Ibarra-Coria), guevaraa@uaeh.edu.mx (Alfredo Guevara-Lara),
} nieto@uaeh.edu.mx (Silvia Nieto-Velázquez) 
Los grupos funcionales son los responsables de las propiedades ácidas de estos compuestos cuyo hidrógeno es susceptible a las reacciones de sustitución. Entre los diversos cationes fijados por el complejo absorbente está el protón $\left(\mathrm{H}^{+}\right)$, es decir, la acidez o reacción de la materia orgánica viene determinada en su mayor parte por la cantidad de cationes hidrógeno fijados en relación con los demás iones, actuando como reguladores de $\mathrm{pH}$ (el pH de los suelos varía entre 5.5 y 8.5, siendo el $\mathrm{pH}$ ideal para un suelo entre 6.0 y 7.5). Por otro lado, entre los factores que afectan la estabilidad de la estructura del húmico en solución acuosa se puede citar el $\mathrm{pH}$, el cual está relacionado con el grado de disociación de los protones de los grupos funcionales (ácidos carboxilos e hidroxilos fenólicos principalmente) aumentando la polaridad del material y su gran capacidad de absorción para compuestos hidrofílicos e hidrófobicos (Chan and Chan, 2003; Lead et al., 2003).

El protón de los grupos carboxilatos (R-COOH) se disocia a valores de $\mathrm{pH}$ entre 4-5, a pH entre 8-9 se disocian los protones de los grupos -OH del fenol y es posible también la disociación de grupos: $-\mathrm{OH}$ enólico, la amida $(=\mathrm{NH})$ y de otros grupos funcionales, los cuales, contribuyen produciendo entre un 85 y 90\% de la carga negativa del humus, considerando a los AHs como ácidos orgánicos de débil disociación. Adicionalmente los grupos protonados como $\left(\mathrm{R}-\mathrm{OH}_{2}\right)^{+}$y $\left(\mathrm{R}-\mathrm{NH}_{3}\right)^{+}$, pueden producir cargas positivas, pero la carga global que permanece y predomina es negativa (Stevenson, 1994). Para estabilizar la carga negativa de los AHs, deben interaccionar con cationes formando complejos estables, modificando la movilidad y fijación de los iones principalmente en los suelos (aumentando su fertilidad) y en agua (procesos de purificación). Tales procesos de formación de cargas negativas pueden explicar el incremento de la capacidad de intercambio cationico (CIC), debido a la disociación de los protones de los grupos funcionales carboxílicos (-COOH) e hidróxidos fenólicos (-OH) principalmente. No obstante, en la actualidad se han realizado diferentes estudios sobre la interacción de los AHs con iones metálicos, obteniendo una mayor información del comportamiento estructural y la reactividad que estas macromoléculas presentan en sistemas ambientales (suelo y agua).

En relación con las características que presenta la MO en específico los AHs como los componentes principales, es analizar las posibles interacciones $A H-X-A l$ ( $\mathrm{X}=$ colorante, agroquímico, As, etc.) de estos compuestos con metales y/o sustancias tóxicas, posteriormente agregar un agente coagulante como son las sales de aluminio ( $\mathrm{Al}$ ) para poder tratar el agua contaminada siendo un recurso natural muy valioso en el mundo, dando un relevante impacto sobre la vida humana, animal, vegetal y de sobrevivencia en todos los aspectos de vida (Kloster et al., 2013a; Watson et al., 2016a). El proceso que recibe el agua residual para ser potabilizada es a través de diversos tratamientos: primario, secundario y terciario. El tratamiento primario consiste especificamente en operaciones de filtración y sedimentación para remover sólidos de elevado tamaño y materia no disuelta. El tratamiento secundario incluye la descomposición de la materia orgánica por la acción de microorganismos y el tratamiento terciario incluye la desinfección del agua mediante técnicas avanzadas (intercambio iónico, adsorción, microfiltración y ultrafiltración, ósmosis inversa, y desindección). El agua resultante de este proceso puede utilizarse para diferentes propósitos domésticos e industriales (Troconis, 2010).

No obstante, el segundo tratamiento todavía lo realizan con agentes coagulantes como son las sales de aluminio, sin embargo, este método es un poco obsoleto, debido principalmente a la contaminación que presenta con el metal, sin embargo, para poder continuar con este tipo de tratamiento para la descontaminación de agua, que consiste en aumentar la efectividad de la remoción de los AHs como MO existente en el medio y sustancias tóxicas que forman como compuestos organoclorados, se plantea una metodología enfocada principalmente a utilizar una concentración mínima pero efectiva del agente coagulante (Al), así como encontrar el pH y las cinéticas de interacción adecuadas (He et al., 2007; Ma et al., 2018).

Es por ello que en este trabajo de investigación se pretende conocer y describir de forma más detallada las propiedades fisicoquímicas y estructurales que presentan los AHs en estudio (comerciales y extraídos en el laboratorio), mediante la correlación y comparación de todos los resultados que se obtengan en la caracterización de estos ácidos. Así como evaluar la agregación de estos compuestos en presencia del catión $\mathrm{Al}$ a diferentes concentraciones (1, 2 y $3 \mathrm{mM}$ ) e intervalos de $\mathrm{pH}$ (5 y 7). Es importante mencionar, que los reportes científicos acerca de las investigaciones de los AHs se han enfocado prácticamente en su estructura, a los mecanismos de reacción (con metales, xenobióticos, halógenos y elementos radioactivos), propiedades ácido-base, propiedades óxido-reducción, entre otros. Sin embargo, estos reportes no son completamente claros a la vez que no hacen mención acerca de un hecho fundamental, la baja estabilidad que presentan en solución acuosa y el resultado que esto conlleva al momento de su análisis, son escasos los artículos que presentan un estudio completo acerca del efecto que presentan con diversas variables (temperatura, radiación, aire, $\mathrm{pH}$ y el método de extracción) sobre la estabilidad; y cómo juegan un papel importante en la interacción con especies contaminantes (Leggett and McBryde, 1975; Kucerík et al., 2004; Nieto, 2010a).

\section{Desarrollo Experimental}

\subsection{Obtención y preparación de la muestra de suelo}

Para la obtención de las muestras de suelo se identificaron lugares no contaminados en relación con lo alejados de la civilización, lo que le llaman un suelo cero, que fueran lugares boscosos con una constante degradación y transformación de la biomasa vegetal y animal (Schnitzer, 1991; Schulten and Hempfling, 1992). No obstante, para la recolección de las muestras nos basamos en la observación del color que presentan: café obscuro o negro, lo cual indica una mayor cantidad de MO (Kononova, 1982a).

Se obtuvo aproximadamente $1 \mathrm{~kg}$ de muestra de cada uno de los suelos (Atotonilco y Tulancingo) del estado de Hidalgo, para la extracción de los AHs se obtuvo el suelo escarbando un promedio de 15 a $20 \mathrm{~cm}$ de profundidad en la capa arable u horizonte A. Posteriormente, a las muestras se les eliminó basura, piedras y raíces, se colocaron en charolas de aluminio para secar en un horno convencional a una temperatura de $60^{\circ} \mathrm{C}$ durante $2 \mathrm{~h}$ (equipo LINDBERG, modelo GO1310A-1), finalmente se tamizaron hasta obtener un tamaño de partícula de $1.0 \mathrm{~mm}$ y se colocaron en recipientes de vidrio y plástico completamente limpios y herméticos.

\subsection{Método de extracción de los AHs}

En la extracción y purificación de los AHs se utilizó el método de Kononova (1982), con ciertas modificaciones con respecto a la separación de los AHs por centrifugación (Kononova, 1982b; Carpio, 2017a). Es un método no agresivo, rápido, económico y 
eficiente, sin embargo, se sugiere utilizar muestras de suelos con una alta cantidad de MO.

Para la extracción de los AHs se pesaron 15 g de suelo y se transfirieron a un matraz Erlenmeyer con capacidad de $250 \mathrm{~mL}$. Posteriormente, se agregaron $120 \mathrm{~mL}$ de una solución recién preparada de $\mathrm{Na}_{2} \mathrm{P}_{2} \mathrm{O}_{7} .10 \mathrm{H}_{2} \mathrm{O}$ con $\mathrm{NaOH}$ ambos reactivos marca SIGMA-ALDRICH con una pureza de $\geq 99 \%$ y $\geq 98 \%$ (11.15 g de $\mathrm{Na}_{2} \mathrm{P}_{2} \mathrm{O}_{7} .10 \mathrm{H}_{2} \mathrm{O}$ y $1 \mathrm{~g}$ de $\mathrm{NaOH}$ en $250 \mathrm{~mL}$ de solución) a $\mathrm{pH} \approx$ 13; el matraz se cerró perfectamente con una septa para evitar la entrada de $\mathrm{CO}_{2}$ y se mantuvo en agitación durante $30 \mathrm{~min}$, se dejó reposar aproximadamente $24 \mathrm{~h}$. Finalmente se filtró utilizando papel filtro whatman No. 42. Al filtrado obtenido se le añadió aproximadamente $2.5 \mathrm{~mL}$ de $\mathrm{HCl}$ concentrado marca BAYER con un 36.5-38\% de pureza, se agitó cuidadosamente y se dejó reposar hasta observar la precipitación de AH. La mezcla se centrifugó por 10 min a 2300 rpm, desechando el sobrenadante y así continuando la operación hasta tener una parte mínima de líquido, prosiguiendo con el filtrado a vacío utilizando membranas Millipore de tipo GVHP $0.22 \mu \mathrm{m}$. El sólido se secó en la estufa a una temperatura de $60{ }^{\circ} \mathrm{C}$ durante $2 \mathrm{~h}$ aproximadamente; se retiró y se colocó en el desecador alrededor de 30 min y finalmente se pesó.

Las muestras obtenidas se preservaron (recipientes color ámbar) para su posterior caracterización con diferentes métodos y técnicas analíticas.

\subsection{Caracterización de los AHs (comercial: AH-CH y extraídos: $A H-A$ y $A H-T)$}

Se trabajó con tres AHs (AH-CH, AH-A y AH-T), considerando que el AH-CH comercial (Chemapex Praha S.R.O.) y los AHs extraídos en el laboratorio AH-A y AH-T fueron caracterizados, evaluados y presentados en trabajos de investigación realizados con anterioridad (Nieto, 2010b; Carpio, 2017b; Nieto, 2017)

Los AHs se caracterizaron por diferentes métodos y técnicas analíticas tanto por vía húmeda e instrumentales, como análisis elemental para la determinación de los componentes elementales como: $\% \mathrm{C}, \% \mathrm{H}, \% \mathrm{~N}$ y por diferencia $\% \mathrm{O}$, por espectroscopia UVVis se obtuvo la absorbancia, concentración, coeficiente de absortividad molar $\varepsilon_{280}$, porcentaje de aromaticidad, peso molecular promedio y el grado de humificación o cociente $\mathrm{E}_{4} / \mathrm{E}_{6}$, en espectroscopia de IRTF se identificaron los grupos funcionales principales: $-\mathrm{COOH},-\mathrm{C}=\mathrm{O},-\mathrm{OH},-\mathrm{CH}_{3}$, entre otros a partir de la obtención de los espectros, en el porcentaje de cenizas se cálculo la cantidad de materia inorgánica disponible y finalmente, la acidez total y cantidad de grupos carboxílicos e hidróxidos fenólicos (-COOH y -OH) (Chin, 1994; Tipping, 2002a; Plaza et al., 2006)

\subsection{Proceso de coagulación de los AHs en presencia de $\mathrm{Al}^{3+}$}

En el proceso de coagulación de los AHs en estudio (AH-CH, $\mathrm{AH}-\mathrm{A}$ y $\mathrm{AH}-\mathrm{T})$, al interaccionar con el metal trivalente $\left(\mathrm{Al}^{3+}\right)$ fue necesario establecer una concentración permisible de AH en las muestras de agua e ir variando la concentración del metal, las soluciones tienen que estar a un $\mathrm{pH}$ establecido, que puede ser de 5 o 7 para evaluar la carga negativa del húmico y la agregación con la carga positiva del metal: $A H-A l$.

\subsubsection{Preparación de soluciones}

Se inició por preparar las soluciones madre de $1000 \mathrm{mg} / \mathrm{l}$ de cada uno de los AHs a partir de 10 mg de húmico en $360 \mu \mathrm{L}$ de
$\mathrm{NaOH} 1 \mathrm{~N}$ y aforando con agua desionizada hasta $10 \mathrm{~mL}$ (Pacheco, 2002), solución madre de $100 \mathrm{mM}$ de $\mathrm{NaCl}$ como electrolito soporte y solución madre de $10 \mathrm{mM}$ de la sal del metal $\left(\mathrm{Al}\left(\mathrm{ClO}_{4}\right)_{3} \cdot 9 \mathrm{H}_{2} \mathrm{O}\right.$, marca SIGMA-ALDRICH con un $98 \%$ de pureza).

\subsubsection{Proceso de coagulación}

Se prepararon un total de cuatro soluciones, la primera solución fue el blanco, que contenía una concentración establecida de 50 mg/l de AH y 15 mM de NaCl marca SIGMA-ALDRICH con un $\geq 99.5 \%$ de pureza como electrolito soporte, las otras tres soluciones presentaban la misma concentración de húmico y electrolito soporte pero se modificó la cantidad del metal, es decir se evaluaron diferentes concentraciones de la sal del metal trivalente a 1, 2 y $3 \mathrm{mM}$ ajustando todas las soluciones a un $\mathrm{pH}$ de 5 , y posteriormente se realizó el mismo procedimiento descrito pero ahora ajustando las soluciones a pH de 7. Se dejaron reposar aproximadamente $16 \mathrm{~h}$ y se obtuvieron las correspondientes lecturas de potencial Zeta $(\zeta)$ utilizando un equipo Malvern modelo Zetasizer nano series (Kloster et al., 2013b; Watson et al., 2016b).

\section{Resultados y Discusión}

El método de extracción que se utiizó resulto ser un tratamiento muy rápido, económico y eficiente comparado con otros métodos más laboriosos, tardados y sobre todo tomado en cuenta un aspecto importante, que son muy drásticos en cuanto a los agentes extractantes, presentando reacciones adversas que modifican la estructura del $\mathrm{AH}$. Sin embargo, es importante tener presente las recomendaciones para la aplicación de éste procedimiento, en relación con no utilizar suelos muy mineralizados y con bajas concentraciones de MO (Licona, 2007).

\subsection{Caracterización de los AHs (AH-CH, AH-A y AH-T)}

Respecto a la caracterización de los AHs resultaron ser ácidos estables y poliácidos con todos los resultados obtenidos por análisis elemental (AE), espectroscopia UV/Vis, cenizas y titulaciones ácido/base (Nieto, 2010c; Carpio, 2017c; Nieto, 2017a) como se presentan en la siguiente tabla:

Tabla 1: Propiedades químicas de los AHs. (Nieto, 2010c; Carpio, 2017c; Nieto, 2017a)

\begin{tabular}{|c|c|c|c|}
\hline \multirow{2}{*}{ Parámetros } & \multicolumn{3}{|c|}{ ÁCIDOS HÚMICOS } \\
\hline & АH-CH & AH-A & AH-T \\
\hline$\% \mathrm{C}$ & $33.67_{(3.14)}$ & $47.38_{(0.96)}$ & $32.31_{(2.77)}$ \\
\hline$\% \mathbf{H}$ & $2.69_{(3.59)}$ & $4.18_{(0.14)}$ & $2.31_{(4.10)}$ \\
\hline$\% \mathbf{N}$ & $0.60_{(0.96)}$ & $2.98_{(2.38)}$ & $0.28_{(6.19)}$ \\
\hline$\% \mathrm{O}^{*}$ & $63.03_{(1.81)}$ & $45.46_{(1.16)}$ & $65.10_{(1.49)}$ \\
\hline \%Cenizas & $11.70(1.00)$ & 6.09 & $50.77_{(1.34)}$ \\
\hline$\varepsilon_{(280)} /\left(\mathrm{L}(\mathrm{mol} \mathrm{de} \mathrm{CO})^{-1} \mathrm{~cm}^{-1}\right)$ & 1409.00 & 1158.01 & 1237.51 \\
\hline \% Aromaticidad & 77.17 & 64.64 & 68.62 \\
\hline P.M. & 6110.32 & 5110.44 & 5427.67 \\
\hline $\mathbf{E}_{4} / \mathbf{E}_{6}$ & 2.07 & 4.26 & 2.06 \\
\hline Acidez total $\left(\right.$ meq g $\left.^{-1}\right)$ & $8.00_{(4.06)}$ & $10.55_{(3.28)}$ & $5.58_{(2.96)}$ \\
\hline $\left.\mathrm{COOH}_{(\text {meq g }} \mathrm{g}^{-1}\right)$ & $3.33_{(1.36)}$ & $4.58_{(3.22)}$ & $1.88_{(13.24)}$ \\
\hline OH (meq g $\left.{ }^{-1}\right)$ & $4.67_{(2.71)}$ & $5.97_{(6.24)}$ & $3.70_{(8.10)}$ \\
\hline
\end{tabular}

\%DER entre paréntesis para un total de seis y tres análisis. Resultados que no reportan $\% \mathrm{DER} \leq 1$. 
Los AHs caracterizados por espectroscopia UV-Vis, presentan espectros muy similares con la misma tendencia (Figura 1), sin embargo, existen pequeñas diferencias en lo que se refiere a las absorbancias correspondientes con base a las diferentes longitudes de onda y en relación a ello diferentes valores en su respectivo cociente: $E_{4} / E_{6}$ (Tabla I). De acuerdo a lo reportado en la literatura (Stevenson, 1994b) los AHs que se analizaron presentan valores del cociente $\mathrm{E}_{4} / \mathrm{E}_{6}<5$, lo cual indica que son ácidos maduros o humificados con mayor carácter aromático y peso molecular promedio, decreciendo en el siguiente orden: $\mathrm{AH}-\mathrm{CH}>$ $\mathrm{AH}-\mathrm{T}>\mathrm{AH}-\mathrm{A}$. Esto último se correlaciona y coincide con el porcentaje de aromaticidad y el peso molecular promedio presentados en la Tabla 1.

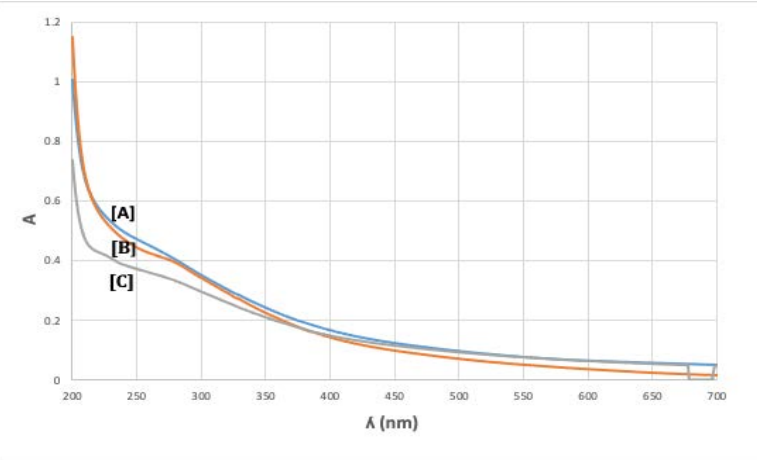

Figura 1: Espectros de absorción UV/Vis: [A] AH-CH, [B] AHA y [C] AH-T, $10 \mathrm{mg} / \mathrm{L}, \mathrm{pH}=11.09$, longitud de celda: $1 \mathrm{~cm}$.

En lo que se refiere a la espectroscopia de IRTF de los AHs (AH-CH, AH-A y AH-T) (Figura 2) revelan la presencia de bandas de absorción muy similares y características, independientemente de su fuente u origen natural de la muestra de suelo, aunque con pequeñas diferencias de intensidad y desplazamientos. Esto permite proponer la presencia de los mismos grupos funcionales principales y más abundantes del tipo $-\mathrm{OH}$, -COOH y -CO, de alcoholes, fenoles, ésteres, cetona o amidas, etc., aunque en diferente proporción.
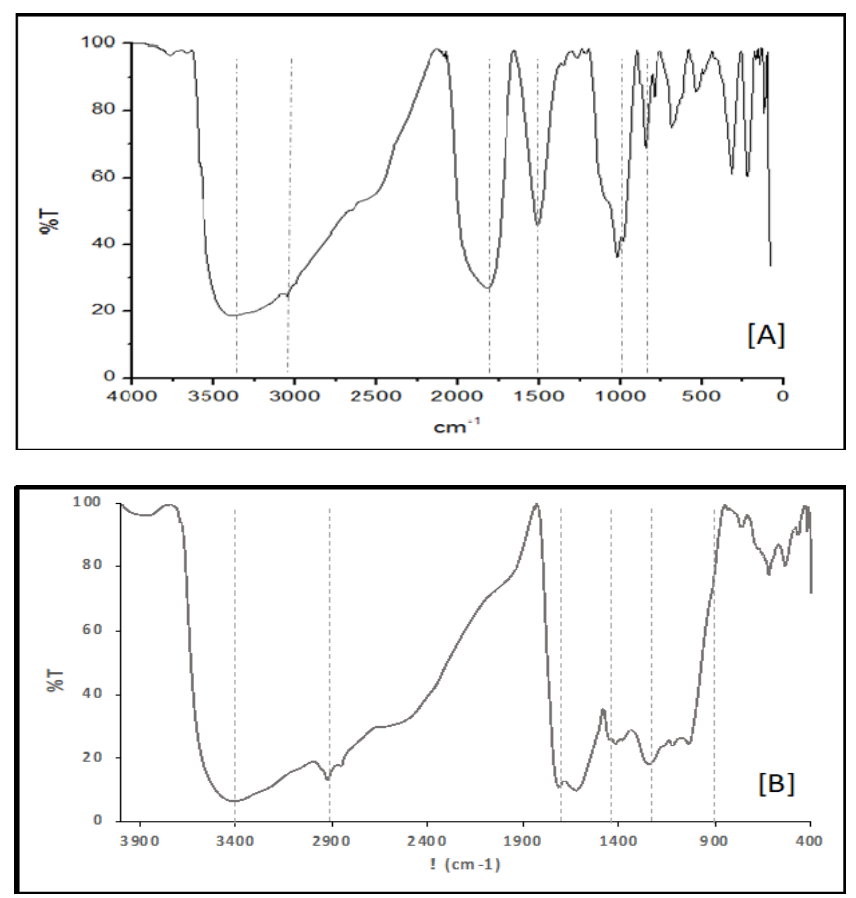

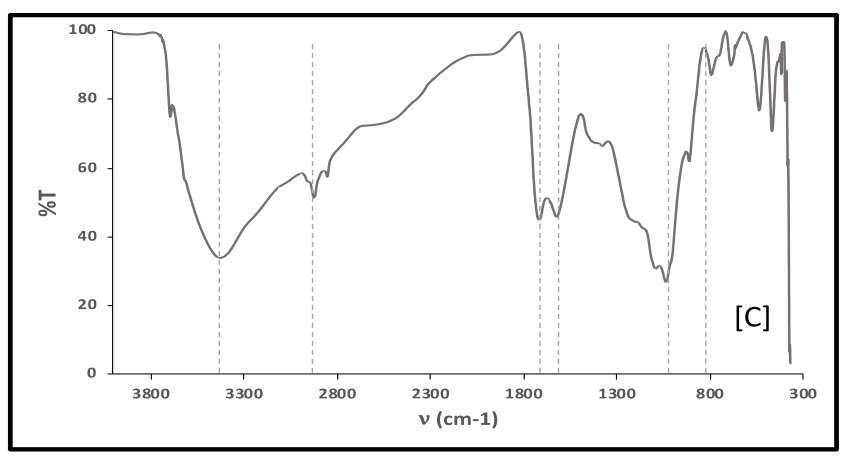

Figura 2: Espectro de IRTF: [A] AH-CH, [B] AH-A y [C] AH-T, analizados en pastilla: con $2.0 \mathrm{mg}$ demuestra en $200 \mathrm{mg}$ de $\mathrm{KBr}$.

En los espectros de IRTF que se presentan en la Figura 2, se observa la presencia de bandas de absorción que caracterizan las vibraciones de alargamiento y de flexión dentro y fuera del plano, así como las huellas dactilares (912 a $450 \mathrm{~cm}^{-1}$ ) en las diferentes agrupaciones atómicas (Tabla 2) (Tipping, 2002b).

Tabla 2: Asignación de las bandas de absorción de los espectros de infrarrojo de la Figura 2

\begin{tabular}{cc}
\hline $\boldsymbol{v}\left(\mathbf{c m}^{-\mathbf{1}}\right)$ & Asignación \\
\hline $3650-3100$ & VO-H, N-H \\
$3100-2840$ & V $-\mathrm{H}$ \\
$1710-1585$ & $\mathrm{~V} \mathrm{C}=\mathrm{O}, \mathrm{C}=\mathrm{C}$ \\
$1440-1330$ & O O-H, $=\mathrm{C}-\mathrm{H}$ \\
& $\mathrm{V}-\mathrm{N}$ \\
$1320-1100$ & $\mathrm{~V}-\mathrm{O}$ \\
& $\mathrm{C}=\mathrm{O}$ \\
$1200-1020$ & VC-C \\
$912-450$ & huellas dactilares \\
\hline
\end{tabular}

En los espectros de IRTF (Figura 2) se observa la presencia de bandas de absorción que caracterizan las vibraciones de alargamiento en las siguientes agrupaciones atómicas: de 3650 a $3100 \mathrm{~cm}^{-1}$ las vibraciones de O-H de alcoholes, fenoles y ácidos carboxílicos, N-H de aminas o amidas; de 3100 a $2840 \mathrm{~cm}^{-1}$ las vibraciones de alargamiento de $\mathrm{C}-\mathrm{H}$ de alcanos, cicloalcanos y alquenos; de 1710-1585 $\mathrm{cm}^{-1}$ las vibraciones de $\mathrm{C}=\mathrm{O}$ que se encuentran en los ácidos carboxílicos, cetonas, ésteres, quinonas y amidas de los compuestos alifáticos y aromáticos; a esta misma región encontramos vibraciones de los dobles enlaces conjugados $\mathrm{C}=\mathrm{C}$, que confirman la presencia de compuestos aromáticos (Rodríguez, 2016).

De esta forma con todos los resultados obtenidos y correlacionando, se pudo coorroborar que el porcentaje de aromaticidad, peso molecular promedio y el grado de humificación es mayor para el AH-CH (comercial) a partir de espectroscopia UV-Vis. Sin embargo, en lo que se refiere a la acidez total de estos compuestos tomando en cuenta las valoraciones acido-base y la señal característica del -OH de grupos funcionales carboxílicos e hidróxidos fenólicos (-COOH y -OH) identificados en los espectros de IRTF es mayor para el AHA. Por otro lado, se observa que el AH-A y AH-CH presentan un mayor porcentaje de hidrógeno y mayor acidez, es decir, tienen una mayor cantidad de grupos funcionales carboxílicos e hidróxidos fenólicos (-COOH y -OH). Sin embargo, de acuerdo a la relación O/C, el AH-T es el que posee mayor cantidad de grupos carboxílicos y/o carbohidratos, pero una menor acidez, lo cual se considera que una parte del oxígeno esté formando complejos con 
metales y/o arcillas, hecho que se refleja en su alto contenido de cenizas.

\subsection{Estudio del proceso de coagulación de los AHs en presencia de $\mathrm{Al}^{3+}$}

El efecto positivo sobre el $\zeta$ o cuando el potencial se vuelve menos negativo de los AHs, debido la presencia del metal o contra-ion, se explica por la reducción de la carga que se unen a los grupos carboxílicos y/o fenólicos negativamente cargados. La magnitud de esta diferencia puede relacionarse también con la afinidad entre la macromolécula y los iones metálicos, en este caso con el correspondiente metal trivalente, quienes presentan una mayor interacción con los grupos carboxílicos (-COO-) y a un pH de 5, tal y como se indica en la literatura (Kloster et al., 2013c; Shunan et al., 2021). Así, es posible ver que los cambios en el valor del $\zeta$ no sólo es el efecto de la modificación en la fuerza iónica de la disolución y, por lo tanto, la compresión de la doble capa que rodea a la macromolécula, sino una medida de la atracción entre AHs y diferentes compuestos iónicos.

Debido a la mínima influencia que tiene la sal de $\mathrm{Na}^{+}$en el potencial zeta del húmico, y con la finalidad de aumentar la conductividad eléctrica de la disolución, a los posteriores análisis se realizaron añadiendo una concentración $15 \mathrm{mM}$ de $\mathrm{NaCl}$ como electrolito soporte. Así, se evaluó bajo estas condiciones el efecto del incremento de la concentración del metal a 1, 2 y $3 \mathrm{mM}$, a valores de pH 5 y 7, y después de un tiempo de 16 h de reacción. Los resultados se observan en las siguientes figuras:

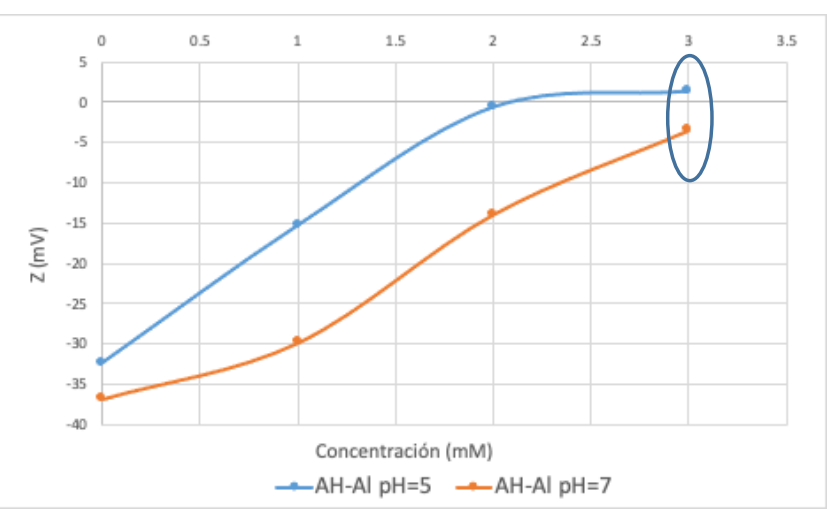

Figura 3: Efecto del $\mathrm{pH}$ en el $\zeta$ del AH-CH en presencia de $\mathrm{Al}^{3+}$ a diferentes concentraciones: 1, 2 y $3 \mathrm{mM}$.

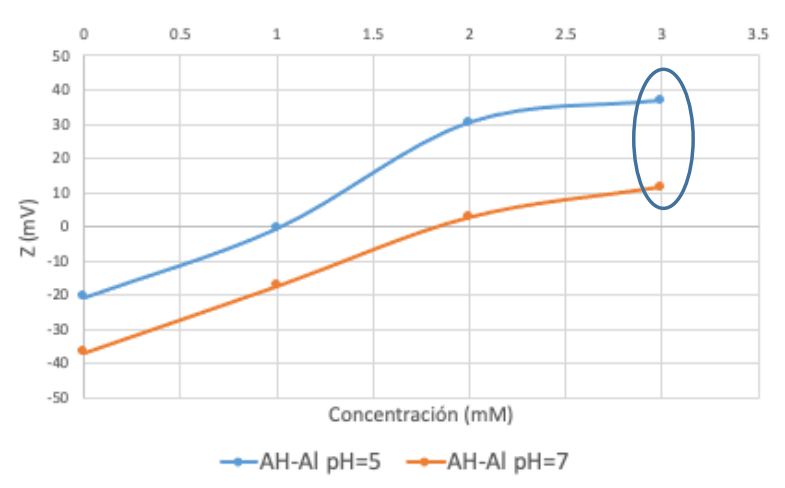

Figura 4: Efecto del $\mathrm{pH}$ en el $\zeta$ del $\mathrm{AH}-\mathrm{A}$ en presencia de $\mathrm{Al}^{3+}$ a diferentes concentraciones: 1 , 2 y 3 mM.

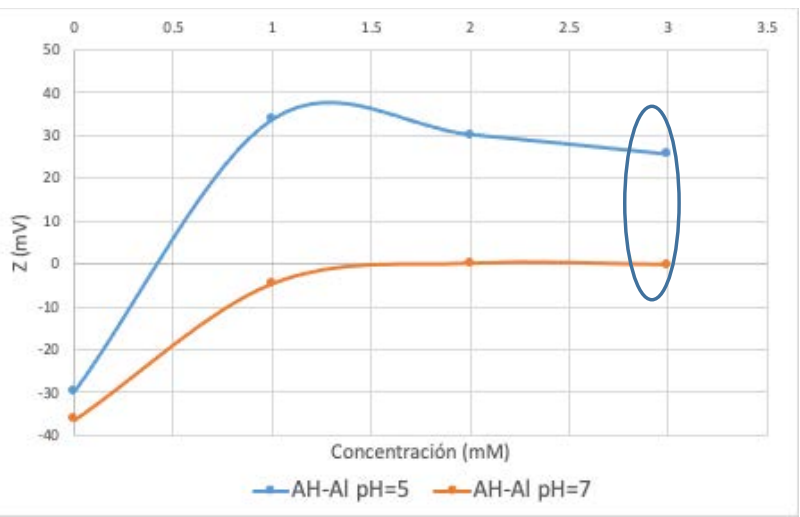

Figura 5: Efecto del $\mathrm{pH}$ en el $\zeta$ del AH-T en presencia de $\mathrm{Al}^{3+}$ a diferentes concentraciones: 1 , 2 y 3 mM.

En la Tabla 3 se presentan los valores promedio del $\zeta$ de los AHs con electrolito soporte $(\mathrm{NaCl})$ en ausencia del metal (soluciones blanco). Se puede observar que su $\zeta$ es independiente del $\mathrm{pH}$ para el AH-CH pero al comparar los valores para el AH-A y AH-T, se tiene una disminución del potencial a pH de 7, es decir valores más negativos. Sin embargo, el efecto de la variación de la concentración (1, 2 y 3 mM) del metal trivalente y del pH (5 y 7), mostró que los valores promedio del $\zeta$ de los mismos AHs si influye y la interacción AH-Metal aumenta a mayor concentración y a un $\mathrm{pH}$ de 5 , se observa como los resultados se vuelven menos negativos o positivos (Tabla 4).

Tabla 3: Valores del $\zeta$ de los AHs a $\mathrm{pH}$ de 5 y 7

\begin{tabular}{ccc}
\hline \multirow{2}{*}{ Muestra } & \multicolumn{2}{c}{ Potencial Zeta $(\boldsymbol{\zeta})$} \\
\cline { 2 - 3 } & $\mathbf{p H}=\mathbf{5}$ & $\mathbf{p H}=\mathbf{7}$ \\
\hline AH-CH & $-32.3(5.14)$ & $-36.8(3.69)$ \\
AH-A & $-20.7(4.89)$ & $-40.8(3.53)$ \\
AH-T & $-29.8(3.52)$ & $-36.2(3.87)$ \\
\hline \multicolumn{2}{c}{ En paréntesis, \%DER de tres análisis }
\end{tabular}

Tabla 4: Valores del $\zeta$ de los $\mathrm{AHs}$ con $\mathrm{Al}^{3+}$ a pH 5 y 7

\begin{tabular}{|c|c|c|c|}
\hline \multirow{3}{*}{ Muestras } & \multicolumn{3}{|c|}{ Potencial Zeta $(\zeta)$} \\
\hline & \multicolumn{3}{|c|}{$\mathrm{pH}=5$} \\
\hline & $1 \mathrm{mM}$ & $2 \mathrm{mM}$ & $3 \mathrm{mM}$ \\
\hline AH-CH-AI & $-15.2(1.38)$ & $-0.55(2.63)$ & $1.41(2.55)$ \\
\hline AH-A-Al & $-0.50(4.59)$ & $30.5(4.30)$ & $37.0(7.99)$ \\
\hline AH-T-Al & $33.7(5.71)$ & $30.1(5.59)$ & $25.5(4.41)$ \\
\hline Muestras & \multicolumn{3}{|c|}{$\mathrm{pH}=7$} \\
\hline AH-CH-Al & $-29.8(4.67)$ & -13.9 & $-3.52(2.75)$ \\
\hline AH-A-Al & $-17.5(0.45)$ & $2.60(1.50)$ & $11.4(5.55)$ \\
\hline AH-T-Al & $-4.67(5.08)$ & $0.03(1.38)$ & $-0.27(4.38)$ \\
\hline
\end{tabular}

En paréntesis, \%DER de tres análisis. Resultados que no reportan \%DER $\leq 1$

Se observa un proceso de agregación y cuagulación, que se ve favorecido para el AH-A al aumentar la concentración del metal y en comparación con el AH-T. Si correlacionamos estos resultados con la señal característica y definida de los ácidos carboxílicos ($\mathrm{COOH}$ ) en el espectro de IRTF podemos de alguna forma precisar que estos ácidos presenta mayor capacidad de interacción o capacidad de intercambio catiónico. 


\section{Conclusiones}

Con el método de extracción utilizando con $\mathrm{Na}_{2} \mathrm{P}_{2} \mathrm{O}_{7} \cdot 10 \mathrm{H}_{2} \mathrm{O}$ e $\mathrm{NaOH}$ como agentes extractantes y mediante la caracterización con los métodos espectroscópicos, análisis elemental, determinación de cenizas y la ácidez total, se obtuvieron en general AHs poliácidos de alto peso molecular promedio, aromaticidad y humificación. No obstante, presentan una gran capacidad de interacción con $\mathrm{Al}^{3+}$ al incrementar la concentración del metal a $3 \mathrm{mM}$ y llevando a cabo el proceso de coagulación, favoreciendo dicho proceso a pH de 5, provocando la disminución de la carga neta negativa de la macromolécula y permitiendo así que el contraión estabilice la carga del húmico formando agregados o aglomerados y logrando la precipitación de los AHs como materia orgánica.

\section{Agradecimientos}

Silvia Nieto Velázquez agradece el financiamiento PRODEP, convenio no. 511-6/18-8644.

\section{Conflicto de intereses}

Los autores declaran que no existen conflictos de intereses.

\section{Referencias}

Aguilera, H. M., (1989). Tratado de edafología de México. 1ª Ed. México, D.F.: Facultad de Ciencias.

Almendros, G. (2008). Revisión analítica de sustancias húmicas en suelos y compost. soilACE 239-255. Recuperado el 2 de mayo de 2018 de: https://www.soilace.com/pdf/pon2008/d26/Cas/04 GAlmendros.pdf.

Carpio, D. K. (2017). Análisis fisicoquímico de ácidos húmicos y evaluación cinética de su agregación con iones $\mathrm{Na}^{+}, \mathrm{Ca}^{2+}$ y $\mathrm{Mg}^{2+}$. Tesis de Licenciatura, Universidad Autónoma del Estado de Hidalgo, México.

Chan, M. N., Chan, C. K. (2003). Hygroscopic properties of two model humiclike substances and their mixtures with inorganics of atmospheric importance. Environ. Sci. Technol 37(22), 5109-5115.

Chin, Y. P., Alken, G., Loughlin, E. O. (1994). Molecular weight, polydispersity, and spectroscopic properties of aquatic humic substances. Environ. Sci. Technol 28, $1853-1858$.

Diallo, M. S., Simpson, A., Gassman, P., Jonson, J. H., Goddard, W. A. (2003). 5.D. Structural modeling of humic acids through experimental characterization, computer assidted structure elucidation and atomistic simulations. 1. Chelsea soil humic acid. Environ. Sci. Technol 37, 17831793.

He, Z., Traina, S. J., Weavers, L. (2007). Sonolytic desorption of mercury from aluminum oxide: effects of $\mathrm{pH}$, chloride and organic matter. Environ. Sci. Technol 41, 779-784.

Kloster, N., Brigante, M., Zanini, G., Avena, M., (2013). Aggregation kinetics of humic acids in the presence of calcium ions. Colloids and Surfaces A: Physicochem. Eng. Aspects 427, 76 - 82.
Kononova, M. M. (1982). Materia orgánica del suelo. $1^{\text {a }}$ Ed. Oikos-tau, Barcelona, España, 63-105.

Kucerík, J., Kovár, J., Pekar, M. (2004). Thermoanalytical investigation of lignite humic acids fractions. J. of Termal Análisis and Calorimetry 76, 6242-6252.

Lead, J. R., Starchev, K., Wilkinson, K. J. (2003). Diffusion coefficients of humic substances in agarose gel and in water. Environ. Sci.Technol 37(3), 482-487.

Leggett, D. J., McBryde, W. A. E. (1975). General computer program for the computation of stability constants from absorbance data. Analytical Chemistry 47(7), 1065-1070.

Licona, S. T. J. (2007). Extracción y análisis fisicoquímico de ácidos húmicos y su interacción con $\mathrm{Cu}(\mathrm{II})$ y $\mathrm{Pb}$ (II). Tesis de Licenciatura, Universidad Autónoma del Estado de Hidalgo, México.

Ma, J., Guo, H., Weng, L., Li, Y., Lei, M., Chen, Y., (2018). Distinct effect of humic acid on ferrihydrite colloid-facilitated transport of arsenic in saturated media at different $\mathrm{pH}$. Chemosphere 212, 794-801.

Mosquera, C., Bravo, I., Hansen, E. (2007). Comportamiento estructural de los ácidos húmicos obtenidos de un suelo andisol del departamento del cauca. Revista Colombiana de Química 36(1), 31-41.

Nieto, V. S. (2010). Estabilidad de los ácidos húmicos y su influencia en la interacción con $\mathrm{Cu}(\mathrm{II})$ y $\mathrm{Pb}(\mathrm{II})$. Tesis de Doctorado, Universidad Autónoma del Estado de Hidalgo, México.

Nieto, V. S. (2017). Evaluación del efecto de las cinéticas de agregación de los ácidos húmicos (AHs) en presencia de $\mathrm{Na}^{+}, \mathrm{Ca}^{2+} \mathrm{y} \mathrm{Mg}^{2+}$. Revista Tendencias en Docencia e Investigación en Química 3, 154 - 162.

Novák, J., Kozler, J., Janos, P., Ceziková, J., Tokarová, V. \& Madronová, L. (2001). Humic acid from coals of the North-Bohemian coal filed I.Preparation and characterization. React. Funct. Polym. 47:101-109.

Pacheco, M. L. (2002). Capillary Electrophoresis and MALDI-TOF mass spectrometry of humic acids. Tesis de Doctorado, Departamento de Química Analítica, Facultad de Ciencias, Universidad de Masaryk, República Checa.

Plaza, C., Brunetti, G., Senesi, N., Polo, A., (2006). Molecular and quantitative analysis of metal ion binding to humic acids from sewage sludge and sludge-amended soils by fluorescence spectroscopy. Environ. Sci. Technol 40(3), 917 - 923.

Rodríguez, W., García, P. A., Fajardo, A. (2016). Aplicaciones de técnicas espectroscópicas para el análisis de suelos. Universidad Militar Nueva Granada 12(2), 228-251.

Schnitzer, M. (1991). Soil organic matter. Soil Sci. 151, 41 - 58.

Schulten, H. R., Hempfling, R. (1992). Influence of agricultural and management on humus compositon and dynamics: Classical and modern analytical techniques. Plant Soil 142, $259-271$.

Shunan, D., Wangwei, C., Jihong, X., Liting, S., Weimu, W., Hui, L. (2021). Aggregation kinetics of fragmental PET nanoplastics in aqueous environment: complex roles of electrolytes, $\mathrm{pH}$ and humic acid. Environmental Pollution 268.

Stevenson, F. J., (1994). Humus chemistry. Genesis, composition and reactions. $2^{\text {nd }}$ Ed. Willey Interscience, New York, NY.

Tipping, E. (2002). Cation binding by humic substances. West Nyack, N.Y. USA: Cambridge University Press. 2002.

Troconis, A. (2010). Tratamiento de aguas residuales. Recuperado 14/05/2021 https://www.belzona.com/es/solution_maps/wastewater/mone y_map.pdf

Watson, M. A., Tubi'c, A., Agbaba, J., Niki'c, J., Maleti'c, S., Molnar, Jazi'c, J., Dalmacija, B., (2016). Response surface methodology investigation into the interactions between arsenic and humic acid in water during the coagulation process. J. Hazardous Materials 312, 150 -158. 